\title{
An obstacle in policymaking and evidence-based planning for enhancing education access to girls-A Comprehensive Analysis of Astonishing School Enrollment Case in Sindh-Pakistan
}

\author{
Mr. Sheraz Ali \\ Senior Manager, Sindh Education-MIS, Reform Support Unit, \\ School Education and Literacy Department, \\ Government of Sindh, Pakistan. \\ Prof. Dr. Syed Abdul Sattar Shah \\ Director - Institute of Business Administration, \\ University of Sindh Jamshoro. \\ Mr. Muhammed Nawaz Sohoo \\ Director of General Mining and Minerals Development Department, \\ Government of Sindh Karachi Pakistan.
}

\author{
Mr. Abdul Karim Tagar \\ Research Associate (MS Fellow), \\ University of Sindh Jamshoro, Sindh Pakistan. \\ Dr. Hamzo Khan Tagar \\ Director Public Private Partnership Node, \\ School Education \& Literacy Department, \\ Government of Sindh, Karachi Pakistan.
}

\begin{abstract}
This Paper has been written in the above context to critically evaluate the Sindh school education enrollment data as a case study in details and suggests recommendation for improvement in data collections system particularly for policy planners in the greater public interest. It is scientific scrutiny of the data and valid for the researchers, decision-makers, development partners including donors and general readers as food for thought and net addition in the available literature on enrollment in the public sector schools.
\end{abstract}

Keywords: Astonishing Data, Annual School Census (ASC), Sindh Education Management Information System (SEMIS) School Enrollments, Statistical Errors, Sustainable Policy, Data Anomalies.

\section{INTRODUCTION}

Sindh Education Management Information System (SEMIS) is collecting the school's enrollment census data annually since 1991 . The annual census provides information about public sector schools running under School Education and Literacy Department (SELD) in three major areas; (i) Enrollment (ii) Human Resource information. (iii) Physical Infrastructure. A typical census form carries 240 fields (30 Questions). The core indicators from the aforementioned three areas are (1) Number of Rooms indicator from physical infrastructure, (2) Enrollment and (3) number of Teachers indicator from Human Resource information area. The researcher is 
convinced that the study "Why policies and practices adopted to enhance girls education in Sindh have not seen fruition" will be a breakthrough in gender disparity as previous studies. "Statistical data regarding various sectors plays a pivotal role in the socio-economic development of a country as the resource allocations are always based on this. Unfortunately, in Pakistan, national figures are usually doubtful and a source of confusion...It is interesting to note that all sources contradict each other. The 'Economic Surveys' and 'Education Policies' which are considered to be the most authentic government documents, both differ from each other." [1].

National Education Policy (NEP) 2017 reveals that "Pre-primary education (3 - 4+ year age group) net enrollment rate (NER) in Pakistan is assumed to be around 36\%, however, no data about NER at early childhood education (ECE) level is available" [2]. On the other hand, there is a huge disconnect between Pakistan's socio-economic indicators and growth, observed, "Pakistan has had respectable per capita growth over 1950-1999, intensive involvement by donors and international agencies ( $\$ 58$ billion in foreign aid)", despite considerable economic growth, country underperformed on the social indicators, like "...Infant mortality and female primary and secondary enrollment are among the worst in the world in Pakistan." furthermore, he stated "Pakistan is also more corrupt, more politically unstable and violent, less respectful of human rights, and less democratic than the benchmark for its level of income" therefore, he quoted "growth without development" [3].

It is important to mention that this paper is based on the PhD Proposal of the lead author [4].

\section{OBJECTIVES}

The reliable, updated and accurate data helps in planning and budgeting. The (SEMIS) data is also used by development partners to design interventions and programs. The purpose of this attempt is to present an analysis of extreme cases where fluctuations, variations, and patterns points to inconsistent data reporting anomalies. It is expected that this study will contribute to setting new benchmarks for the data verification process in the Annual School Census (ASC), exercise.

The specific objectives of the study were to:

I. An evaluation of the Sindh school education enrollment data.

II. Assess the details of data in public interest.

III. Examine different aspects of data, its bottlenecks and feasible recommendations for producing quality data for sustainable policy and evidence based planning.

\section{RESEARCH QUESTIONS}

The study was guided by the following questions:

I. How have Sindh Education Management Information's system collected data for planning?

II. What is the quality of the data provided by institutions of the province?

III. What is its impact on policy making and evidence-based planning for enhancing education access to all particularly on girls.

\section{METHODOLOGY\& DATA}

The study sorted biased and unbiased statically errors in the data in the school education system of Pakistan and findings of the study can be used by the policymakers to improve the data collection and managing it in decent manners. The author's experiences are incorporated at large. The qualitative and quantitative strategy has been adopted to identify the relevant issue in Sindh School's education system in public interest and in systemically order. 


\section{DISCUSSION AND RESULTS}

A. Extreme Enrollment Cases: it is based on thorough data analysis techniques, the following cases are identified using standard data query techniques. In the first case, the same enrollment reported in both years which is less possible. The first criteria identify schools that are reporting exactly the same enrollment numbers class-wise in annual school census (ASCs). There are 374 such schools having 31,012 enrollments. Further discussion on such schools are observed in following sections of the report as mentioned in

Table -01

\begin{tabular}{|c|ccccccccccc|c|c|c|c|c|}
\hline Year & K & C 1 & C 2 & C 3 & C 4 & C 5 & C 6 & C 7 & C 8 & C 9 & C 10 & C 11 & C 12 & Total \\
\hline $\mathbf{0 1}$ & 200 & 100 & 95 & 90 & 85 & 80 & 75 & 70 & 65 & 60 & 55 & 50 & 45 & 1070 \\
\hline $\mathbf{0 2}$ & 200 & 100 & 95 & 90 & 85 & 80 & 75 & 70 & 65 & 60 & 55 & 50 & 45 & 1070 \\
\hline $\begin{array}{c}\text { Diff. } \\
\text { \% }\end{array}$ & $\mathbf{0}$ & $\mathbf{0}$ & $\mathbf{0}$ & $\mathbf{0}$ & $\mathbf{0}$ & $\mathbf{0}$ & $\mathbf{0}$ & $\mathbf{0}$ & $\mathbf{0}$ & $\mathbf{0}$ & $\mathbf{0}$ & $\mathbf{0}$ & $\mathbf{0}$ & $\mathbf{0}$ \\
\hline
\end{tabular}

K=Kachi Class, $\boldsymbol{C}=$ Class, Year 01=Previous Year (2012-13), Year 02=Current Year (2013-14)

B. Fluctuated enrollment case: In Criteria, for case ' $B$ ' the schools reported current academic year's enrollment in annual school census (ASC). The current enrollment sometimes is higher or lower than the previous year depending on factors such as intake in grades, dropped out children and promotion to the next grade. Under this criterion, schools are identified which are outliers in terms of significant fluctuations in two academic years which is also not properly justified in our analysis of the data as mentioned in table 02.

Table -02

\begin{tabular}{|cccccccccccccc|c|c|c|}
\hline Year & K & C 1 & C 2 & C 3 & C 4 & C 5 & C 6 & C 7 & C 8 & C 9 & C 10 & C 11 & C 12 & Total \\
\hline $\mathbf{0 1}$ & 200 & 100 & 95 & 90 & 85 & 800 & 75 & 70 & 65 & 60 & 55 & 50 & 45 & 1790 \\
\hline $\mathbf{0 2}$ & 100 & 0 & 95 & 90 & 85 & 80 & 75 & 70 & 65 & 60 & 55 & 50 & 45 & 870 \\
\hline $\begin{array}{c}\text { Diff. } \\
\text { \% }\end{array}$ & NA & NA & NA & NA & NA & NA & NA & NA & NA & NA & NA & NA & NA & $\mathbf{- 6 9}$ \\
\hline
\end{tabular}

$\boldsymbol{K}=$ Kachi Class, $\boldsymbol{C}=$ =lass, Year 01=Previous Year (2012-13), Year 02=Current Year (2013-14), NA=Not Applicable

C. Fluctuated Enrollment of a specific class: In the analysis of case ' $C$ ' in detail, other criteria used are high enrollment grades within the school. Under this criterion for case "C" the school's enrollment of Year 02 (ASC 2013-14) is more than 50 and enrollment of specific class enrollment is more than or equal to $50 \%$ as resulted in table 03 which is also less possible in the field.

Table-3

\begin{tabular}{|c|c|c|c|c|c|c|c|c|c|c|c|c|c|c|}
\hline Year & K & C 1 & C 2 & C 3 & C 4 & C 5 & C 6 & C 7 & C 8 & C 9 & C 10 & C 11 & C 12 & Total \\
\hline $\mathbf{0 1}$ & NA & NA & NA & NA & NA & NA & NA & NA & NA & NA & NA & NA & NA & NA \\
\hline $\mathbf{0 2}$ & 10 & 10 & 10 & 10 & 100 & 10 & 0 & 0 & 0 & 0 & 0 & 0 & 0 & 150 \\
\hline $\begin{array}{c}\text { Enrol. \% } \\
\text { of Specific } \\
\text { Class }\end{array}$ & 6.6 & 6.6 & 6.6 & 6.6 & $\mathbf{6 6 . 6 7}$ & 6.6 & 0 & 0 & 0 & 0 & 0 & 0 & 0 & 100 \\
\hline
\end{tabular}

$\boldsymbol{K}=$ Kachi Class, $\boldsymbol{C}=$ Class, Year 01=Previous Year (2012-13), Year 02=Current Year (2013-14), $N A=$ Not Applicable 
D. High dropout and unrealistic promotion rates: The promotion rates of two academic years are analyzed. The assumption is that promotion from grade $\mathrm{x}$ to grade $\mathrm{x}+1$ must have some consistency. For example, it seems unlikely that a cohort of 200 pupils in class-I would increase to 400 pupils in class-II in the next academic year. An illustration is provided below. Schools enrollment of Year 2 (Annual School Census 2013-14) is more than 200 and Students promoted from class-I (year 01) to I+1 (Year 02) are Less than and equal to 25\%, OR more than or equal to 200\% (excluding campus Schools and entrance classes i.e Kachi, Class 1, 6, 9 and 11), see below example in table 04 which also reflects an astonishing case of enrollment in the schools' data.

\begin{tabular}{|c|c|c|c|c|c|c|c|c|c|c|c|c|c|c|}
\hline Year & K & C 1 & C 2 & C 3 & C 4 & C 5 & C 6 & C 7 & C 8 & C 9 & C 10 & C 11 & C 12 & Total \\
\hline $\mathbf{0 1}$ & 200 & $\mathbf{2 0 0}$ & 95 & 90 & 85 & 80 & 75 & 80 & 65 & 60 & 55 & 50 & 45 & 1070 \\
\hline $\mathbf{0 2}$ & 200 & 175 & 400 & 125 & 90 & 85 & 60 & 65 & 20 & 40 & 45 & 30 & 30 & 1265 \\
\hline $\begin{array}{l}\text { Promotion } \\
\text { Rate }\end{array}$ & NA & NA & $\mathbf{2 0 0}$ & 132 & 100 & 100 & NA & 87 & $\mathbf{2 5}$ & NA & 75 & NA & 60 & 118 \\
\hline
\end{tabular}

E. Astonishing Enrollment Cases Result and others Anomalies in the districts: The first criterion identifies schools that are reporting exactly the same enrollment numbers class-wise. suspicious enrollment was observed in 374 schools $(0.8 \%)$ and top one district was Tharparkar therein 31 schools class wise enrollment reported exactly the same as reported in the previous year which is not reliable data as resulted below and it proved the case of astonishing enrollment case in Sindh province schools which affects largely on policy planning and access to quality education for all because of satirical errors in the data.

Table -05

\begin{tabular}{|c|l|c|}
\hline S.No & \multicolumn{1}{|c|}{ District Name } & $\begin{array}{c}\text { \# of } \\
\text { Schools }\end{array}$ \\
\hline 1 & Tharparkar & 31 \\
\hline 2 & Kashmore & 25 \\
\hline 3 & Sujawal & 25 \\
\hline 4 & Khairpur Mirs & 24 \\
\hline 5 & Thatta & 23 \\
\hline 6 & Dadu & 21 \\
\hline 7 & Umerkot & 21 \\
\hline 8 & Sanghar & 20 \\
\hline 9 & Mirpur Khas & 19 \\
\hline 10 & Tando Muhammad Khan & 16 \\
\hline 11 & Jacobabad & 15 \\
\hline 12 & Kambar-Shahdadkot & 14 \\
\hline 13 & Jamshoro & 13 \\
\hline 14 & Naushero Feroze & 12 \\
\hline 15 & Central Karachi & 11 \\
\hline
\end{tabular}

\begin{tabular}{|c|c|c|}
\hline S.No & District Name & $\begin{array}{c}\text { \# of } \\
\text { Schools } \\
\end{array}$ \\
\hline 16 & Ghotki & 11 \\
\hline 17 & Badin & 9 \\
\hline 18 & Larkana & 9 \\
\hline 19 & Mitiari & 9 \\
\hline 20 & South Karachi & 9 \\
\hline 21 & Sukkur & 9 \\
\hline 22 & Tando Allah Yar & 6 \\
\hline 23 & Hyderabad & 5 \\
\hline 24 & Korangi Karachi & 4 \\
\hline 25 & Shikarpur & 4 \\
\hline 26 & East Karachi & 3 \\
\hline 27 & Malir Karachi & 3 \\
\hline 28 & West Karachi & 2 \\
\hline 29 & Shaheed Benazirabad & 1 \\
\hline & Total & 374 \\
\hline
\end{tabular}

F. Anomalies in case B: Schools enrollment of Year 2 (ASC 2013-14) is more than 50 and the difference between year 1 and Year 2 enrollment is more than $150 \%$ or Less than $-50 \%$ (excluding campus schools).The highly fluctuated enrollment reported in 468 (1\%) schools, where the top schools are mentioned below and the top district was Dadu, where 60 schools found in a highly fluctuated category and these fluctuations and astonishing create obstacles in 
Ali, S., Shah, S. A. S., Sohoo, M. N., Tagar, A. K., \& Tagar, H. K. (2020). An obstacle in policymaking and evidence-based planning for enhancing education access to girls-A Comprehensive Analysis of Astonishing School Enrollment Case in Sindh-Pakistan. Advances in Social Sciences Research Journal, 7(2) 166-180.

policy planning in Sindh Pakistan and harmful for real gender enrollments data in long term planning for women's literacy and empowerments in Sindh Pakistan. For more details see table 06, 07 and 08 given below.

Table -06

District Sujawal, 404070067 GGPS HUSSAIN DAL @ GOOGANI YOON (-137\% fluctuation observed)

\begin{tabular}{|c|c|c|c|c|c|c|c|c|c|c|c|c|c|c|c|c|}
\hline $\begin{array}{l}\text { ASC } \\
\text { Year }\end{array}$ & $\begin{array}{l}\ddot{Z} \\
\widetilde{U} \\
\widetilde{y}\end{array}$ & $\begin{array}{l}\vec{y} \\
\tilde{y} \\
\frac{\pi}{v}\end{array}$ & $\begin{array}{l}N \\
\tilde{n} \\
\tilde{\pi} \\
\tilde{U}\end{array}$ & $\begin{array}{l}m \\
w \\
\tilde{\pi} \\
\tilde{\sigma}\end{array}$ & 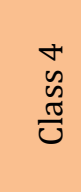 & $\begin{array}{l}10 \\
\tilde{y} \\
\tilde{\pi} \\
\tilde{U}\end{array}$ & $\begin{array}{l}0 \\
\text { 足 } \\
\tilde{\pi} \\
\vec{U}\end{array}$ & $\begin{array}{l}\hat{y} \\
y \\
\tilde{d} \\
0\end{array}$ & $\begin{array}{l}\infty \\
0 \\
0 \\
\frac{\pi}{U}\end{array}$ & $\begin{array}{c}\sigma \\
\tilde{y} \\
\frac{\pi}{v}\end{array}$ & 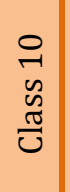 & $\begin{array}{l}\vec{y} \\
\tilde{n} \\
\tilde{\sigma} \\
\vec{v}\end{array}$ & 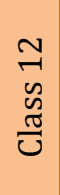 & 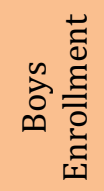 & 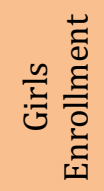 & 胥 \\
\hline Year 01 & 16 & 132 & 102 & 86 & 140 & 47 & 0 & 0 & 0 & 0 & 0 & 0 & 0 & 0 & 523 & 523 \\
\hline Year 02 & 0 & 23 & 16 & 21 & 15 & 22 & 0 & 0 & 0 & 0 & 0 & 0 & 0 & 0 & 97 & 97 \\
\hline
\end{tabular}

Table -07

District Badin, 401040305 GBPS ALI MUHAMMAD KHANGHAR@MINRO KOLHI (186\% fluctuation observed)

\begin{tabular}{|c|c|c|c|c|c|c|c|c|c|c|c|c|c|c|c|c|}
\hline ASC Year & 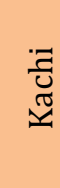 & $\begin{array}{l}\vec{r} \\
\tilde{w} \\
\frac{\pi}{U}\end{array}$ & $\begin{array}{l}N \\
\tilde{w} \\
\tilde{\sigma} \\
\tilde{U}\end{array}$ & $\begin{array}{l}m \\
\text { nd } \\
\tilde{d} \\
\tilde{U}\end{array}$ & $\begin{array}{l}\dot{y} \\
\tilde{D} \\
\tilde{\sigma} \\
v\end{array}$ & $\begin{array}{l}n \\
\tilde{n} \\
\tilde{\pi} \\
\tilde{U}\end{array}$ & $\begin{array}{l}0 \\
\tilde{W} \\
\tilde{\pi} \\
0\end{array}$ & $\begin{array}{l}\hat{n} \\
\tilde{n} \\
\tilde{v}\end{array}$ & $\begin{array}{l}\infty \\
\tilde{n} \\
\tilde{\sigma} \\
\tilde{U}\end{array}$ & $\begin{array}{l}a \\
\tilde{y} \\
\tilde{n} \\
\tilde{U}\end{array}$ & 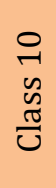 & $\begin{array}{l}\vec{J} \\
\tilde{w} \\
\tilde{\sigma}\end{array}$ & $\begin{array}{l}\sim \\
\tilde{w} \\
\tilde{\sigma} \\
\tilde{U}\end{array}$ & 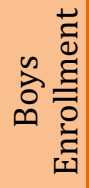 & 出 & 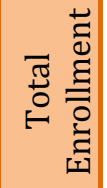 \\
\hline Year 01 & 2 & 0 & 0 & 0 & 0 & 0 & 0 & 0 & 0 & 0 & 0 & 0 & 0 & 1 & 1 & 2 \\
\hline Year 02 & 12 & 25 & 20 & 0 & 0 & 0 & 0 & 0 & 0 & 0 & 0 & 0 & 0 & 41 & 16 & 57 \\
\hline
\end{tabular}

Table-08

'Case B - Categories of Highly Fluctuated Enrollment'

Fluctuated Enrollment Category

Number of

Schools

For all schools reported in year two having more than 50 enrollment and not declared as campus school and Enrollment difference between year 1 and Year 2 is more than $150 \%$ or Less than $-50 \%$

For all schools reported in year two having less than or equal to 50 enrollment and difference between year 1 and Year 2 is more than $150 \%$ or Less than $-50 \%$

Enrollment difference between year 1 and Year 2 is between $101 \%$ to $150 \%$ or Between $26 \%$ to $-50 \%$

Enrollment difference between year 1 and Year 2 is between $51 \%$ to $100 \%$ or Between $-16 \%$ to $-25 \%$

Enrollment difference between year 1 and Year 2 is between $26 \%$ to $50 \%$ or Between $-6 \%$ to $15 \%$

Remaining Schools
468

5,204

4,246

4,634

8,444

23728 
Table 09 - District wise summary of case B Fluctuated enrollment

\begin{tabular}{|c|l|c|}
\hline S.No & \multicolumn{1}{|c|}{ District Name } & \# of Schools \\
\hline 1 & Dadu & 60 \\
\hline 2 & Jacobabad & 49 \\
\hline 3 & Kambar-Shahdadkot & 46 \\
\hline 4 & Tharparkar & 31 \\
\hline 5 & Kashmore & 26 \\
\hline 6 & Korangi Karachi & 24 \\
\hline 7 & Badin & 23 \\
\hline 8 & Sujawal & 19 \\
\hline 9 & Sanghar & 17 \\
\hline 10 & West Karachi & 16 \\
\hline 11 & Thatta & 14 \\
\hline 12 & South Karachi & 14 \\
\hline 13 & Khairpur Mirs & 13 \\
\hline 14 & Central Karachi & 12 \\
\hline 15 & Malir Karachi & 12 \\
\hline
\end{tabular}

\begin{tabular}{|c|l|c|}
\hline S.No & \multicolumn{1}{|c|}{ District Name } & \# of Schools \\
\hline 16 & Naushero Feroze & 11 \\
\hline 17 & Jamshoro & 10 \\
\hline 18 & East Karachi & 9 \\
\hline 19 & Ghotki & 8 \\
\hline 20 & Shaheed Benazirabad & 8 \\
\hline 21 & Tando Muhammad Khan & 7 \\
\hline 22 & Larkana & 7 \\
\hline 23 & Umerkot & 6 \\
\hline 24 & Hyderabad & 6 \\
\hline 25 & Mirpur Khas & 5 \\
\hline 26 & Tando Allah Yar & 5 \\
\hline 27 & Shikarpur & 3 \\
\hline 28 & SUKKUR & $\mathbf{4 6 8}$ \\
\hline 29 & Mitiari & \\
\hline & & Total
\end{tabular}

G. Anomalies analysis in case C: Enrollment of a specific class is more than or equal to the $50 \%$ of overall enrollment was observed in 1,696 (3.6\%) schools, in other words, it was high fluctuation in enrollment of a particular class. Moreover in primary classes high fluctuated enrollment found in a school located in Jacobabad, similarly, the high fluctuated enrollment is observed in the post-primary school located in Karachi Gadap Town. It is evident that district Naushero Feroze is the top one district where 165 schools reported with high fluctuation in a particular class. For detail see table 10,11and 12 given below.

Table-10 .District Jacobabad, 412020266 GGPS QADIR PUR - II ( CMS )

\begin{tabular}{|c|c|c|c|c|c|c|c|c|c|c|c|c|c|c|c|}
\hline $\begin{array}{l}\ddot{\bar{U}} \\
\mathbb{\mathscr { I }}\end{array}$ & $\begin{array}{l}\vec{y} \\
\tilde{\sigma} \\
\vec{v}\end{array}$ & $\begin{array}{l}N \\
w \\
\tilde{w} \\
\tilde{U}\end{array}$ & $\begin{array}{l}m \\
w \\
\tilde{J} \\
\tilde{U}\end{array}$ & $\begin{array}{l}+ \\
\tilde{y} \\
\tilde{\sigma} \\
\end{array}$ & 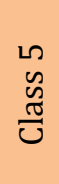 & $\begin{array}{l}0 \\
\tilde{w} \\
\tilde{U} \\
v\end{array}$ & $\begin{array}{l}\hat{y} \\
\tilde{\sigma} \\
\tilde{U}\end{array}$ & 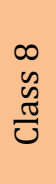 & $\begin{array}{l}a \\
\tilde{y} \\
\tilde{w} \\
\tilde{U}\end{array}$ & 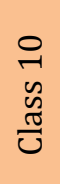 & $\begin{array}{l}\vec{z} \\
\tilde{w} \\
\tilde{w} \\
\vec{v}\end{array}$ & \begin{tabular}{l}
\multirow{1}{*}{} \\
$\tilde{y}$ \\
$\frac{\pi}{U}$
\end{tabular} & 总莺 & 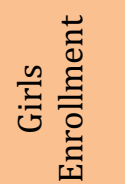 & 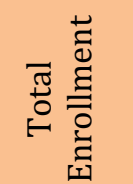 \\
\hline 331 & 40 & 36 & 23 & 18 & 9 & 0 & 0 & 0 & 0 & 0 & 0 & 0 & 0 & 457 & 457 \\
\hline
\end{tabular}

Table-11.Malir Karachi (Gadap Town) 408180466 GBHSS HIGHER SECONDARY SCHOOL

\begin{tabular}{|c|c|c|c|c|c|c|c|c|c|c|c|c|c|c|c|}
\hline & & & & & & & & & & & & & & & \\
\hline $\begin{array}{l}\tilde{\Xi} \\
\tilde{\Psi} \\
\tilde{\Psi}\end{array}$ & $\begin{array}{l}-\vec{y} \\
\tilde{y} \\
\tilde{\sigma} \\
v\end{array}$ & $\begin{array}{l}N \\
\tilde{n} \\
\tilde{\pi} \\
0\end{array}$ & $\begin{array}{l}m \\
\tilde{n} \\
\tilde{n} \\
\tilde{U}\end{array}$ & $\begin{array}{l}+ \\
\tilde{y} \\
\tilde{\sigma} \\
\tilde{U}\end{array}$ & $\begin{array}{l}n \\
\tilde{n} \\
\tilde{\pi} \\
\tilde{U}\end{array}$ & $\begin{array}{l}0 \\
\text { ஸे } \\
\tilde{\pi} \\
\tilde{U}\end{array}$ & $\begin{array}{l}\hat{y} \\
y \\
\tilde{y} \\
\tilde{U}\end{array}$ & $\begin{array}{l}\infty \\
0 \\
\tilde{w} \\
\tilde{\sigma}\end{array}$ & $\begin{array}{l}a \\
\text { y } \\
\tilde{\sigma} \\
0\end{array}$ & $\begin{array}{l}0 \\
\stackrel{1}{0} \\
\tilde{w} \\
\stackrel{0}{U}\end{array}$ & 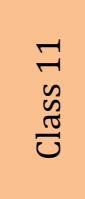 & $\begin{array}{l}\mathcal{N} \\
\tilde{y} \\
\tilde{y} \\
\tilde{U}\end{array}$ & 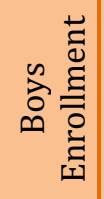 & 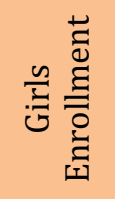 & 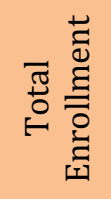 \\
\hline 0 & 0 & 0 & 0 & 0 & 0 & 7 & 7 & 5 & 65 & 135 & 115 & 349 & 465 & 218 & 683 \\
\hline
\end{tabular}


Ali, S., Shah, S. A. S., Sohoo, M. N., Tagar, A. K., \& Tagar, H. K. (2020). An obstacle in policymaking and evidence-based planning for enhancing education access to girls-A Comprehensive Analysis of Astonishing School Enrollment Case in Sindh-Pakistan. Advances in Social Sciences Research Journal, 7(2) 166-180.

Table-12: District wise summary from Case C: Fluctuate Enrollment of a specific class (Any class enrollment is more than or equal to $50 \%$ of overall enrollment)

\begin{tabular}{|c|c|c|}
\hline S.No & District Name & \# of Schools \\
\hline 1 & Naushero Feroze & 165 \\
\hline 2 & Shaheed Benazirabad & 129 \\
\hline 3 & Dadu & 117 \\
\hline 4 & Khairpur Mirs & 115 \\
\hline 5 & Jacobabad & 114 \\
\hline 6 & Badin & 113 \\
\hline 7 & Ghotki & 96 \\
\hline 8 & Sujawal & 90 \\
\hline 9 & Sanghar & 89 \\
\hline 10 & Kambar-Shahdadkot & 81 \\
\hline 11 & Tharparkar & 81 \\
\hline 12 & Thatta & 76 \\
\hline 13 & Mirpur Khas & 56 \\
\hline 14 & Umerkot & 50 \\
\hline 15 & Tando Allah Yar & 47 \\
\hline
\end{tabular}

\begin{tabular}{|l|l|c|}
\hline S.No & \multicolumn{1}{|c|}{ District Name } & $\begin{array}{c}\text { \# of } \\
\text { Schools }\end{array}$ \\
\hline 16 & Shikarpur & 41 \\
\hline 17 & SUKKUR & 36 \\
\hline 18 & Hyderabad & 34 \\
\hline 19 & Malir Karachi & 32 \\
\hline 20 & Larkana & 23 \\
\hline 21 & Tando Muhammad Khan & 20 \\
\hline 22 & South Karachi & 18 \\
\hline 23 & Jamshoro & 18 \\
\hline 24 & Mitiari & 15 \\
\hline 25 & West Karachi & 10 \\
\hline 26 & Kashmore & 696 \\
\hline 27 & Central Karachi & 11 \\
\hline 28 & Korangi Karachi & 10 \\
\hline 29 & East Karachi & \\
\hline Total & & 18 \\
\hline
\end{tabular}

H. Anomalies Analysis in case D: Schools enrollment of Year 2 (ASC 2013-14) is more than 200 and Students promoted from class-I (year 01) to I+1 (Year 02) are less than and equal to $25 \%$ OR more than or equal to $200 \%$ (excluding campus Schools and entrance classes i.e Kachi, Class 1, 6, 9 and 11), This is very tricky formula (detail is given 3.4 heading) the School in District Ghotki, GBPS IRRIGATION COLONY (SEMIS ID 419010214), in ASC 2012-13 Class 1 total 90 students enrolled and the ASC 2013-14 in Class 2 total 211 (Promotion rate is 234\%), the similar unrealistic promotion rates and high dropout is observed in $182(0.4 \%)$. Schools and the top district was Naushero Feroze where 25 schools have high fluctuation in promotion or dropout rates. For detail see table 13. 
Table 13- District wise summary from Case D: High dropout and unrealistic promotion rates

\begin{tabular}{|c|l|c|}
\hline S.No & \multicolumn{1}{|c|}{ District Name } & \# of Schools \\
\hline 1 & Naushero Feroze & 25 \\
\hline 2 & Shaheed Benazirabad & 20 \\
\hline 3 & Dadu & 19 \\
\hline 4 & Khairpur Mirs & 18 \\
\hline 5 & Jacobabad & 13 \\
\hline 6 & Badin & 9 \\
\hline 7 & Ghotki & 8 \\
\hline 8 & Sujawal & 7 \\
\hline 9 & Kambar-Shahdadkot & 6 \\
\hline 10 & Sanghar & 6 \\
\hline 11 & Tharparkar & 5 \\
\hline 12 & Thatta & 5 \\
\hline 13 & Mirpur Khas & 4 \\
\hline 14 & Shikarpur & 4 \\
\hline 15 & Tando Allah Yar & \\
\hline
\end{tabular}

\begin{tabular}{|c|l|c|}
\hline S.No & \multicolumn{1}{|c|}{ District Name } & \# of Schools \\
\hline 16 & Umerkot & 4 \\
\hline 17 & Sukkur & 3 \\
\hline 18 & Larkana & 3 \\
\hline 19 & Malir Karachi & 3 \\
\hline 20 & Hyderabad & 3 \\
\hline 21 & Tando Muhammad Khan & 3 \\
\hline 22 & Jamshoro & 2 \\
\hline 23 & South Karachi & 2 \\
\hline 24 & Mitiari & 2 \\
\hline 25 & Kashmore & 1 \\
\hline 26 & West Karachi & 1 \\
\hline 27 & Central Karachi & 0 \\
\hline 28 & Korangi Karachi & 0 \\
\hline 29 & East Karachi & $\mathbf{1 8 2}$ \\
\hline \multicolumn{2}{|l}{} \\
\hline
\end{tabular}

I. Anomalies analysis in case E: It is evident that 3,652 (7.8\%) schools found too low and too high students and teachers ratio (STR) and the top one is Shaheed Benazirabad where 384 Schools observed with too low or too high STR For detail see table 14 below and 15 .

Table -14

\begin{tabular}{|l|c|}
\hline Category & \# of Schools \\
\hline Student Teacher Ratio (STR) Too High & 3,163 \\
\hline Student Teacher Ratio (STR) Too low & 489 \\
\hline
\end{tabular}


Ali, S., Shah, S. A. S., Sohoo, M. N., Tagar, A. K., \& Tagar, H. K. (2020). An obstacle in policymaking and evidence-based planning for enhancing education access to girls-A Comprehensive Analysis of Astonishing School Enrollment Case in Sindh-Pakistan. Advances in Social Sciences Research Journal, 7(2) 166-180.

Table 15- District wise summary from Case E: Student Teacher Ratio (STR) too low OR too high

\begin{tabular}{|c|c|c|c|c|c|}
\hline S.No & District Name & \# of Schools & S.No & District Name & \# of Schools \\
\hline 1 & Shaheed Benazirabad & 384 & 16 & Korangi Karachi & 93 \\
\hline 2 & Ghotki & 291 & 17 & Larkana & 92 \\
\hline 3 & Dadu & 272 & 18 & Jamshoro & 92 \\
\hline 4 & Khairpur Mirs & 267 & 19 & Umerkot & 81 \\
\hline 5 & South Karachi & 217 & 20 & SUKKUR & 70 \\
\hline 6 & Naushero Feroze & 207 & 21 & East Karachi & 65 \\
\hline 7 & Sujawal & 187 & 22 & Hyderabad & 64 \\
\hline 8 & Badin & 184 & 23 & Shikarpur & 53 \\
\hline 9 & Jacobabad & 159 & 24 & Kashmore & 49 \\
\hline 10 & Tharparkar & 144 & 25 & Tando Allah Yar & 42 \\
\hline 11 & Sanghar & 117 & 26 & Mitiari & 35 \\
\hline 12 & Mirpur Khas & 106 & 27 & Tando Muhammad Khan & 33 \\
\hline 13 & West Karachi & 105 & 28 & Central Karachi & 27 \\
\hline 14 & Kambar-Shahdadkot & 102 & 29 & Malir Karachi & 16 \\
\hline 15 & Thatta & 98 & & Total & 3,652 \\
\hline
\end{tabular}

J. Anomalies Analysis in Case F: It is also observed that 3,649 (7.8\%) schools found too low and too high School Class Ratio (SCR) and the top one Dadu where 350 Schools observed with too low or too high school class ratio (SCR) For detail see table 16 given below in district wise summary.

Table 16

\begin{tabular}{|c|l|c|}
\hline S.No & \multicolumn{1}{|c|}{ District Name } & \# of Schools \\
\hline 1 & Dadu & 350 \\
\hline 2 & Kambar-Shahdadkot & 322 \\
\hline 3 & Khairpur Mirs & 273 \\
\hline 4 & Jacobabad & 265 \\
\hline 5 & Larkana & 198 \\
\hline 6 & Ghotki & 195 \\
\hline 7 & Shaheed Benazirabad & 184 \\
\hline 8 & Naushero Feroze & 179 \\
\hline 9 & Sanghar & 157 \\
\hline 10 & Badin & 145 \\
\hline 11 & Kashmore & 142 \\
\hline 12 & Tharparkar & 140 \\
\hline 13 & Shikarpur & 120 \\
\hline 14 & Mirpur Khas & 115 \\
\hline 15 & Sujawal & 104 \\
\hline & & \\
\hline
\end{tabular}

\begin{tabular}{|c|l|c|}
\hline S.No & \multicolumn{1}{|c|}{ District Name } & $\begin{array}{c}\text { \# of } \\
\text { Schools }\end{array}$ \\
\hline 16 & Sukkur & 96 \\
\hline 17 & Umerkot & 85 \\
\hline 18 & Thatta & 83 \\
\hline 19 & Hyderabad & 80 \\
\hline 20 & Tando Allah Yar & 61 \\
\hline 21 & Central Karachi & 59 \\
\hline 22 & Mitiari & 49 \\
\hline 23 & Jamshoro & 47 \\
\hline 24 & Tando Muhammad Khan & 44 \\
\hline 25 & Malir Karachi & 40 \\
\hline 26 & South Karachi & 37 \\
\hline 27 & Korangi Karachi & 36 \\
\hline 28 & West Karachi & 26 \\
\hline 29 & East Karachi & 17 \\
\hline & & $\mathbf{3 , 6 4 9}$ \\
\hline
\end{tabular}

K. Anomalies Analysis in case G: It is observed that between 1 to 10 students reported as per criteria $377(0.8 \%)$ found low enrollment and district Umerkot is top one where 58 Schools reported with low enrollment. For detail see table 17 which shows District wise summary from Case G: School has too low enrollment. 
Table-17

\begin{tabular}{|c|l|c|}
\hline S.No & \multicolumn{1}{|c|}{ District Name } & $\begin{array}{c}\text { \# of } \\
\text { Schools }\end{array}$ \\
\hline 1 & Umerkot & 58 \\
\hline 2 & Thatta & 32 \\
\hline 3 & Sujawal & 29 \\
\hline 4 & Khairpur Mirs & 28 \\
\hline 5 & Badin & 26 \\
\hline 6 & Tharparkar & 24 \\
\hline 7 & Malir Karachi & 24 \\
\hline 8 & Sanghar & 21 \\
\hline 9 & Mirpur Khas & 20 \\
\hline 10 & Central Karachi & 20 \\
\hline 11 & South Karachi & 15 \\
\hline 12 & Mitiari & 11 \\
\hline 13 & Ghotki & 10 \\
\hline 14 & Shaheed Benazirabad & 7 \\
\hline 15 & Tando Muhammad Khan & 7 \\
\hline
\end{tabular}

\begin{tabular}{|c|l|c|}
\hline S.No & \multicolumn{1}{|c|}{ District Name } & $\begin{array}{c}\text { \# of } \\
\text { Schools }\end{array}$ \\
\hline 16 & Naushero Feroze & 6 \\
\hline 17 & Sukkur & 6 \\
\hline 18 & Shikarpur & 4 \\
\hline 19 & Hyderabad & 4 \\
\hline 20 & Tando Allah Yar & 4 \\
\hline 21 & Jamshoro & 4 \\
\hline 22 & Dadu & 3 \\
\hline 23 & Kambar-Shahdadkot & 3 \\
\hline 24 & Jacobabad & 3 \\
\hline 25 & Korangi Karachi & 3 \\
\hline 26 & East Karachi & 2 \\
\hline 27 & Larkana & 1 \\
\hline 28 & Kashmore & 1 \\
\hline 29 & West Karachi & $\mathbf{3 7 7}$ \\
\hline \multicolumn{2}{|l|}{} \\
\hline
\end{tabular}

L. All Cases Result: The individual cases calculation showing total Schools are 10,398, but Combining all Schools selected through Astonishing Enrollment cases labeled from A to G and removing the duplication, only 7,974 schools filtered which is $17 \%$ of overall schools reported in Sindh (Total 46,724 ). District Dadu was the top one where the total 679 Schools found in the anomalies. The enrollment figure of those 7974 schools was observer $1,409,345$ which is $34.5 \%$ of overall enrollment $(4,085,415)$, for detail see table 18,19 and 20 .

Table 18 - Combining all Schools selected through Astonishing Enrollment

\begin{tabular}{|c|c|c|c|c|c|c|c|}
\hline $\begin{array}{c}\text { Case A } \\
\text { Schools }\end{array}$ & $\begin{array}{c}\text { Case B } \\
\text { Schools }\end{array}$ & $\begin{array}{c}\text { Case C } \\
\text { Schools }\end{array}$ & $\begin{array}{c}\text { Case D } \\
\text { Schools }\end{array}$ & $\begin{array}{c}\text { Case E } \\
\text { Schools }\end{array}$ & $\begin{array}{c}\text { Case F } \\
\text { Schools }\end{array}$ & $\begin{array}{c}\text { Case G } \\
\text { Schools }\end{array}$ & $\begin{array}{c}\text { Total } \\
\text { Schools }\end{array}$ \\
\hline 374 & 468 & 1696 & 182 & 3652 & 3649 & 377 & 10398 \\
\hline
\end{tabular}

Table 19- Combining all Schools selected through Astonishing Enrollment

\begin{tabular}{|l|c|}
\hline \multicolumn{1}{|c|}{ Category of School } & \# of Schools \\
\hline Unique Schools (Case A 292+B 333+C 1157+D 63+E 1942+F 1970+G 174) & 5,931 \\
\hline Any two cases Intersect with each other & 1,679 \\
\hline Any three cases Intersect with each other & 347 \\
\hline Any four cases Intersect with each other & 17 \\
\hline
\end{tabular}


Ali, S., Shah, S. A. S., Sohoo, M. N., Tagar, A. K., \& Tagar, H. K. (2020). An obstacle in policymaking and evidence-based planning for enhancing education access to girls-A Comprehensive Analysis of Astonishing School Enrollment Case in Sindh-Pakistan. Advances in Social Sciences Research Journal, 7(2) 166-180.

Table 20- District wise summary from All Case ranging from A to G

\begin{tabular}{|c|c|c|c|c|c|c|c|c|c|c|}
\hline $\begin{array}{l}\text { S. } \\
\text { No }\end{array}$ & District Name & 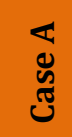 & 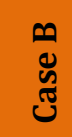 & 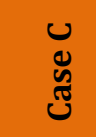 & 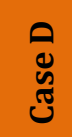 & ㄸ⿱ & 赔 & 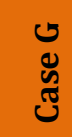 & 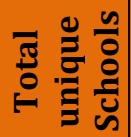 & $\begin{array}{c}\text { Astonishing } \\
\text { Enrollment }\end{array}$ \\
\hline 1 & Dadu & 21 & 60 & 117 & 20 & 384 & 350 & 3 & 679 & 135,601 \\
\hline 2 & Khairpur Mirs & 24 & 13 & 115 & 8 & 291 & 273 & 28 & 579 & 101,247 \\
\hline 3 & Kambar-Shahdadkot & 14 & 46 & 81 & 18 & 267 & 322 & 3 & 528 & 104,401 \\
\hline 4 & Jacobabad & 15 & 49 & 114 & 25 & 207 & 265 & 3 & 500 & 101,792 \\
\hline 5 & Ghotki & 11 & 8 & 96 & 19 & 272 & 195 & 10 & 475 & 85,834 \\
\hline 6 & Naushero Feroze & 12 & 11 & 165 & 7 & 187 & 179 & 6 & 444 & 89,699 \\
\hline 7 & Tharparkar & 31 & 31 & 81 & 2 & 217 & 140 & 24 & 400 & 38,452 \\
\hline 8 & Sanghar & 20 & 17 & 89 & 13 & 159 & 157 & 21 & 396 & 70,942 \\
\hline 9 & $\begin{array}{l}\text { Shaheed } \\
\text { Benazirabad }\end{array}$ & 1 & 8 & 129 & 5 & 144 & 184 & 7 & 377 & 68,518 \\
\hline 10 & Badin & 9 & 23 & 113 & 5 & 98 & 145 & 26 & 322 & 46,265 \\
\hline 11 & Kashmore & 25 & 26 & 10 & 9 & 184 & 142 & 1 & 313 & 57,459 \\
\hline 12 & Larkana & 9 & 7 & 23 & 6 & 102 & 198 & 1 & 299 & 107,250 \\
\hline 13 & Mirpur Khas & 19 & 5 & 56 & 1 & 105 & 115 & 20 & 267 & 40,867 \\
\hline 14 & Sujawal & 25 & 19 & 90 & 0 & 93 & 104 & 29 & 257 & 29,796 \\
\hline 15 & Thatta & 23 & 14 & 76 & 2 & 92 & 83 & 32 & 230 & 21,207 \\
\hline 16 & Shikarpur & 4 & 5 & 41 & 5 & 106 & 120 & 4 & 222 & 48,540 \\
\hline 17 & Umerkot & 21 & 6 & 50 & 0 & 65 & 85 & 58 & 220 & 25,395 \\
\hline 18 & Sukkur & 9 & 3 & 36 & 6 & 117 & 96 & 6 & 215 & 38,580 \\
\hline 19 & Hyderabad & 5 & 6 & 34 & 3 & 70 & 80 & 4 & 169 & 38,890 \\
\hline 20 & Tando Allah Yar & 6 & 5 & 47 & 4 & 81 & 61 & 4 & 154 & 27,219 \\
\hline 21 & Central Karachi & 11 & 12 & 8 & 3 & 92 & 59 & 20 & 144 & 16,762 \\
\hline 22 & Jamshoro & 13 & 10 & 18 & 4 & 53 & 47 & 4 & 118 & 28,544 \\
\hline 23 & South Karachi & 9 & 14 & 18 & 3 & 64 & 37 & 15 & 116 & 8,057 \\
\hline 24 & T. Muhammad Khan & 16 & 7 & 20 & 2 & 35 & 44 & 7 & 111 & 9,961 \\
\hline 25 & Mitiari & 9 & 2 & 15 & 1 & 49 & 49 & 11 & 110 & 19,027 \\
\hline 26 & Malir Karachi & 3 & 12 & 32 & 4 & 42 & 40 & 24 & 108 & 12,385 \\
\hline 27 & Korangi Karachi & 4 & 24 & 6 & 3 & 33 & 36 & 3 & 98 & 17,577 \\
\hline 28 & West Karachi & 2 & 16 & 11 & 3 & 16 & 26 & 1 & 70 & 12,896 \\
\hline 29 & East Karachi & 3 & 9 & 5 & 1 & 27 & 17 & 2 & 53 & 6,182 \\
\hline
\end{tabular}




\section{Interactions between all cases}

\section{i. Interactions between case A with Case B, C, D, E, F \& G}

A notable overlap observed between Case A \& E where 46 schools $(1.2 \%)$ have the same enrollment with low or high student Teacher Ratio (STR) reported in figure 01, however, there is no overlapping between Case A \& B. Moreover, remaining cases overlapping is given below: $\mathrm{A}$ interaction C: 17 Schools $(0.8 \%)$ have same enrollment and specific class fluctuated. A interaction D: Only 1 School $(0.2 \%)$ has the same enrollment and high promotion rates interaction F: 42 Schools (1.1\%) have the same enrollment with Low or High Student Classroom Ratio (SCR) A interaction G:10 Schools (1.3\%) have same enrollment and very low enrollment.

\section{ii. Interactions between case B with Case A, C, D, E, F \& G}

A substantial overlap between Case B \& C where 60 schools (2.9\%) have overall fluctuated enrollment and also specific class fluctuated as shown in figure 02 , and no overlapping between case B \& A and Case G \& G. see below all combinations. Moreover, remaining cases overlapping is given below interaction D: 3 Schools $(0.5 \%)$ have fluctuated enrollment and high promotion or dropout rates' interaction E: 69 Schools (1.7\%) have fluctuated enrollment with Low or High Student Teacher Ratio (STR) B interaction F: 35 Schools (0.9\%) have fluctuated enrollment with Low or High Student Classroom Ratio (SCR)

\section{iii. Interactions between case $C$ with Case $A, B, D, E, F \& G$}

A significant number of schools overlap between Case C \& E where 362 schools (7.3\%) have a specific class fluctuated and low or high student Teacher Ratio (STR) reported in figure 03, and no overlapping between case $\mathrm{C} \& \mathrm{G}$. Moreover, remaining cases overlapping is given below interaction A: 17 Schools (0.8\%) have a specific class fluctuated and same enrollment both years interaction D: 12 Schools $(0.6 \%)$ have a specific class fluctuated and high promotion or dropout rates $\mathrm{C}$ interaction F: 295 Schools (5.8\%) have a specific class fluctuated \& Low or High Student Classroom Ratio (SCR)
Figure-01

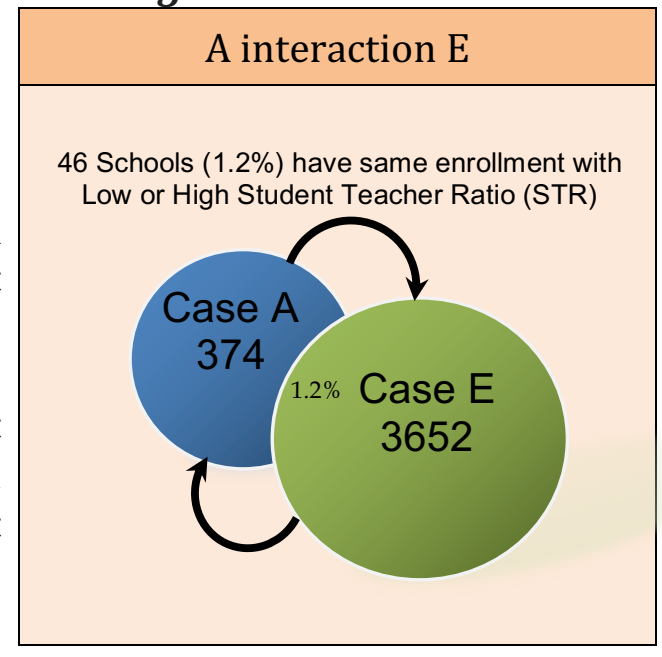

Figure-02

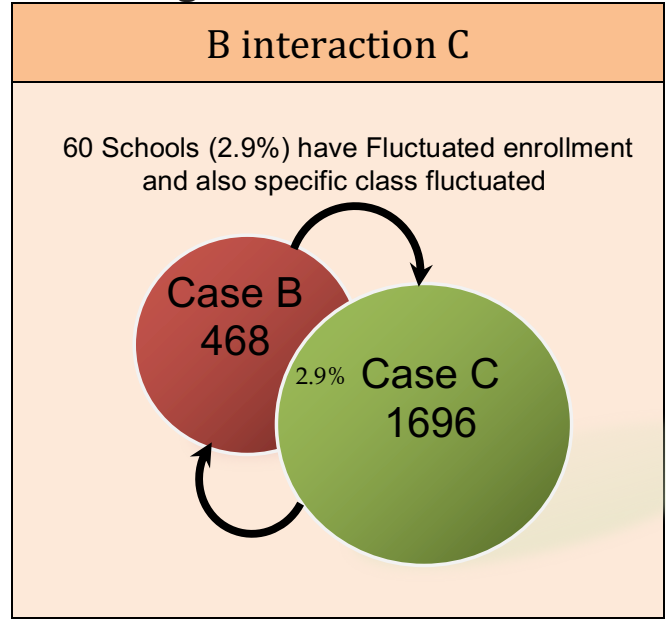

Figure-03

C interaction E

362 Schools $(7.3 \%)$ have a specific class fluctuated \& Low or High Student Teacher Ratio (STR)

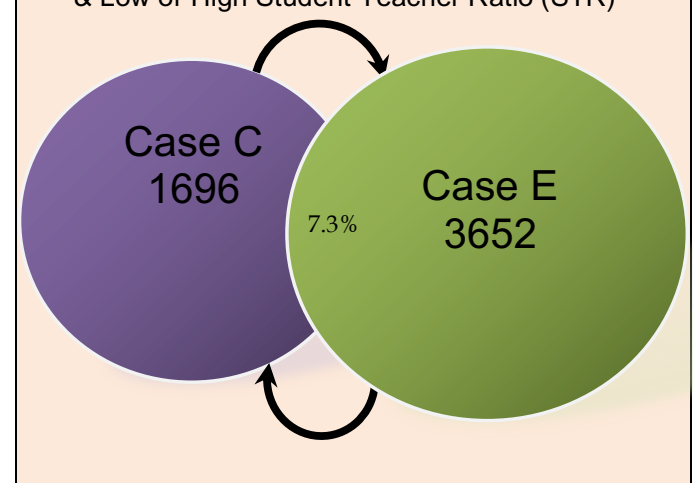


Ali, S., Shah, S. A. S., Sohoo, M. N., Tagar, A. K., \& Tagar, H. K. (2020). An obstacle in policymaking and evidence-based planning for enhancing education access to girls-A Comprehensive Analysis of Astonishing School Enrollment Case in Sindh-Pakistan. Advances in Social Sciences Research Journal, 7(2) 166-180.

iv. Interactions between case D with Case A, B, C, E, F \& G A major overlap between Case E \& F observed where 103 (2.8\%) schools high promotion or dropout rate with Low or High Student Classroom Ratio (SCR) however, there is no interaction between Case D \& G. Moreover, remaining cases overlapping is given below interaction A: Only 1 School $(0.2 \%)$ has high promotion rate and same enrollment with previous Year D interaction B: 3 Schools $(0.5 \%)$ have high promotion rates and enrollment fluctuated interaction C: 12 Schools $(0.6 \%)$ have high promotion or dropout rate and specific class fluctuated interaction E: 51 Schools (1.3\%) have a high promotion or dropout rates with Low or High Student Teacher Ratio (STR). Details are discussed in figures 04 and 05.

\section{Interactions between case $E$ with Case A, B, C, D, F \& G}

A Large number of schools overlap between Case $E$ \& F where 1415 (24.0\%) schools have Low or High Student Teacher Ratio (STR) \& Low or High Student Classroom Ratio (SCR). The low overlap observed 46 Schools (1.2\%) between Case E \& A have Low or High Student Teacher Ratio (STR) \& the same enrollment both years. Moreover, remaining cases overlapping is given below interaction $\mathrm{B}$ : 69 Schools (1.7\%) have Low or High Student Teacher Ratio (STR) and also overall enrollment fluctuated interaction C: 362 Schools (7.3\%) have Low or High Student Teacher Ratio (STR) \& specific class fluctuated interaction D: 51 Schools (1.3\%) have Low or High Student Teacher Ratio (STR) \& high promotion or dropout rates interaction G: 136 Schools (3.5\%) have Low or High STR Low enrollment.

\section{vi. Interactions between case F with Case A, B, C, D, E \& G} A very large number of schools overlap between Case $E$ \& F where 1415 (24.0\%) schools have Low or High Student Teacher Ratio (STR) \& Low or High Student Classroom Ratio (SCR) reflected in the figure 06. The low overlap observed between Case F \& B found where 36 Schools $(0.9 \%)$ have Low or High Student Classroom Ratio (SCR) and overall enrollment fluctuated. Moreover, remaining cases overlapping is given below: $F$ interaction A:43 Schools $(1.1 \%)$ have Low or High Student Classroom Ratio (SCR) and same enrollment both years interaction C:295 Schools (5.8\%) have Low or High Student Classroom Ratio (SCR) \& specific class fluctuated interaction D:103 Schools (2.8\%) have Low or High Student Classroom Ratio (SCR) and high promotion or dropout rates interaction G:164 Schools (4.2\%) have Low or High Student Teacher Ratio (STR) and Low enrollment.

\section{Figure-04}

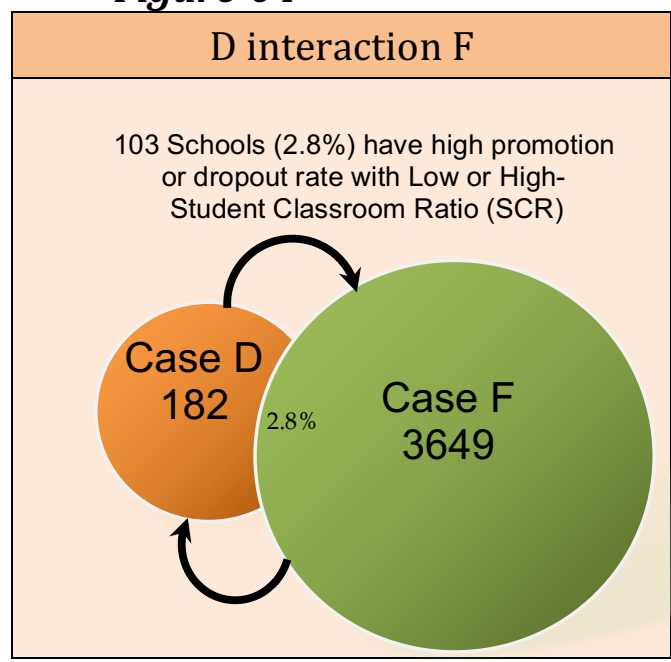

Figure-05

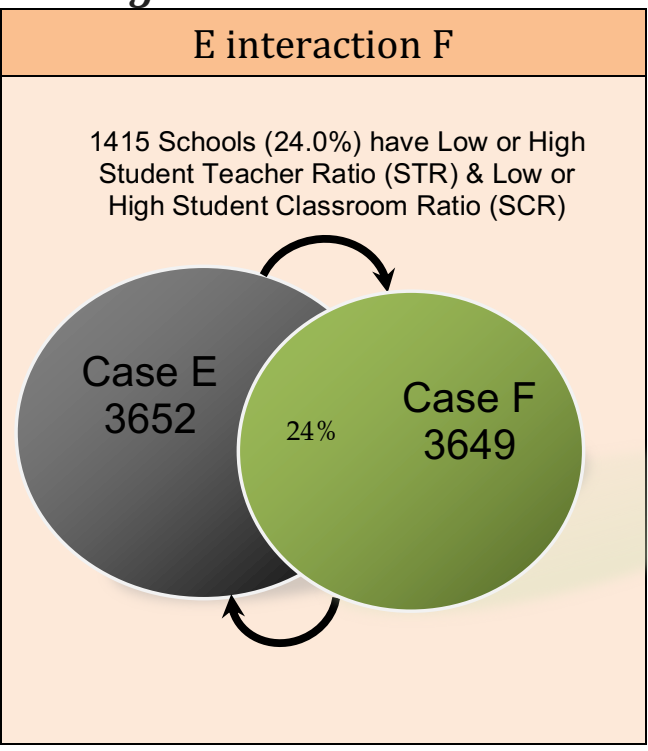

Figure-06

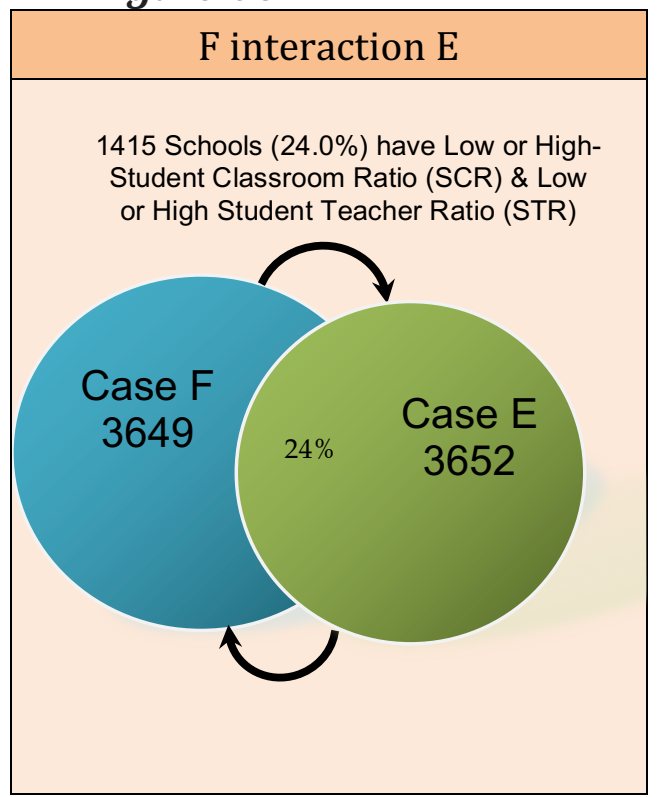


vii. Interactions between case $G$ with Case A, B, C, D, E \& F

A notable overlap found between Case G \& F, where 164 (4.2\%) Schools have Low or High Student Teacher Ratio (STR) and Low enrollment examined in figure 07. There is no overlap between $G$ \& $A, G \& B, G \& C$ and $D \& G$. Moreover, remaining cases overlapping is given below interaction A:10 Schools (1.3\%) have very low enrollment and the same enrollment in both Years G interaction E:136 Schools $(3.5 \%)$ have Low or High Student Teacher Ratio (STR) and Low enrollment. All interactions are elaborated in figures mentioned above for the easy understanding of the readers as in figure 01 to 07 . [5].
Figure-07

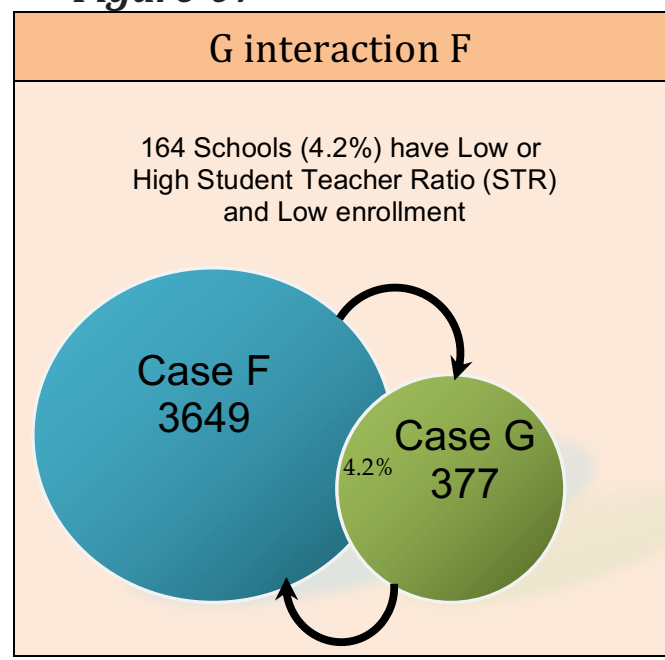

\section{Gender and Level Wise Proportions of Astonishing Enrollment and Challenge of Evidence-Based Planning For Enhancing Education Access to Girls:}

In terms of proportions, as compared to boys more of astonishing enrollment of girls were observed in Karchi (Boys 35.1\% \& Girls 36.8\%) and Primary level classes from Class $1^{\text {st }}$ to class $5^{\text {th }}$ (Boys $28.5 \%$ \& Girls 29.3\%), contrary in post-primary classes (Class $6^{\text {th }}$ to $12^{\text {th }}$ ) more boys were reported, detail calculation is explained in table 21 and figure 08.

\begin{tabular}{|c|c|c|c|c|c|}
\hline 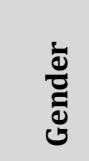 & $\begin{array}{c}\text { Kachi / } \\
\text { ECE }\end{array}$ & $\begin{array}{l}\text { Primary: } \\
\text { Class } \\
1 \text { to } 5\end{array}$ & $\begin{array}{c}\text { Middle: } \\
\text { Class } \\
6 \text { to } 8\end{array}$ & $\begin{array}{l}\text { Second.: } \\
\text { Class } \\
9 \text { to } 10\end{array}$ & $\begin{array}{c}\text { Hi. } \\
\text { Second.: } \\
\text { Class } \\
11 \text { to } 12\end{array}$ \\
\hline \multicolumn{6}{|c|}{ As per Census 2013-14 } \\
\hline Boys & 321,449 & $1,468,134$ & 360,190 & 221,584 & 54,098 \\
\hline Girls & 244,264 & 969,964 & 272,150 & 147,489 & 26,093 \\
\hline Total & 565,713 & $2,438,098$ & 632,340 & 369,073 & 80,191 \\
\hline \multicolumn{6}{|c|}{ Figures \& Percentages form Astonishing cases } \\
\hline \multicolumn{6}{|c|}{ Gender Wise Numbers } \\
\hline Boys & 112,717 & 419,144 & 165,219 & 112,049 & 36,799 \\
\hline Girls & 89,927 & 284,571 & 107,837 & 64,802 & 16,280 \\
\hline Total & 202,644 & 703,715 & 273,056 & 176,851 & 53,079 \\
\hline \multicolumn{6}{|c|}{ Gender Wise Proportion } \\
\hline Boys & $35.1 \%$ & $28.5 \%$ & $45.9 \%$ & $50.6 \%$ & $68.0 \%$ \\
\hline Girls & $36.8 \%$ & $29.3 \%$ & $39.6 \%$ & $43.9 \%$ & $62.4 \%$ \\
\hline Total & $35.8 \%$ & $28.9 \%$ & $43.2 \%$ & $47.9 \%$ & $66.2 \%$ \\
\hline
\end{tabular}

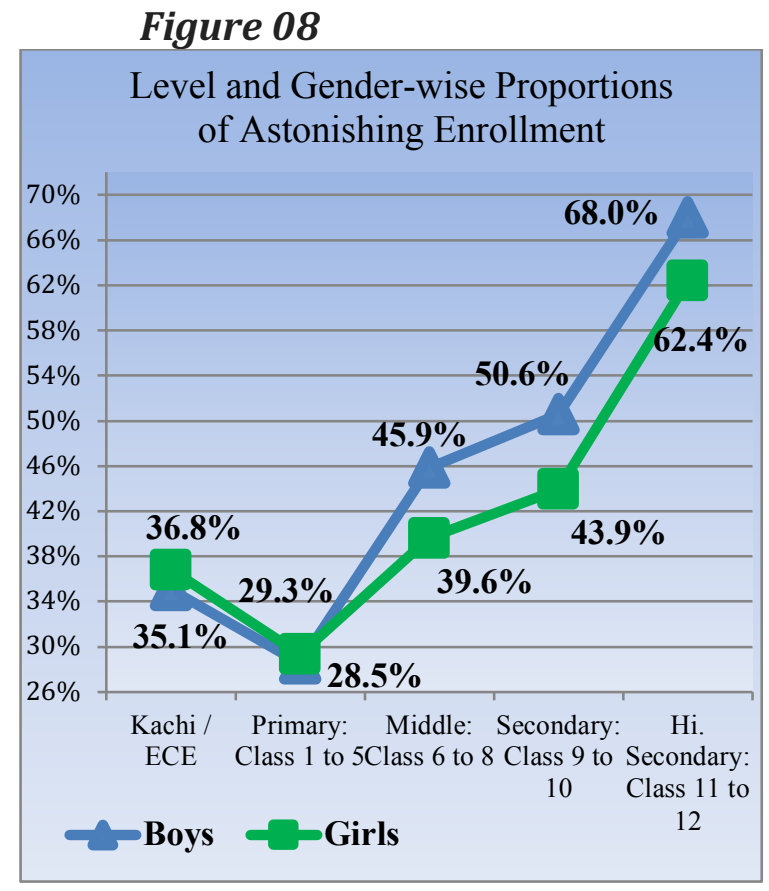

[6].

\section{CONCLUSIONS AND POLICY RECOMMENDATIONS}

It is concluded that the result of the comprehensive analysis shows that out of overall schools 46,724 anomalies in the enrollment data found in 7,974 schools wherein 1,409,345 enrollments reported which is $34.5 \%$ of overall enrollment $(4,085,415)$, now there is a need to further investigate and find out the reasons why such enrollment is available in the system. But it is important to conclude that astonishing enrollments in the schools, large scale fluctuations in the enrollments system, biased and unbiased statistical errors in the data of the 
Ali, S., Shah, S. A. S., Sohoo, M. N., Tagar, A. K., \& Tagar, H. K. (2020). An obstacle in policymaking and evidence-based planning for enhancing education access to girls-A Comprehensive Analysis of Astonishing School Enrollment Case in Sindh-Pakistan. Advances in Social Sciences Research Journal, 7(2) 166-180.

children affected on the literacy trends and quality educations access. its major obstacles in sustainable policy planning for tomorrow's people.

Recommendation: Researcher strongly believed that the role of technology is to facilitate the reform, therefore, a robust integrated centralized system is proposed which not only import data from various sources then assemble the data and generate the anomalies for further checking, correction \& update (if any) but through that progress of all educational indicators, comparisons, tracking \& monitoring can be done easily. In this regard, following points may be useful in the development of such system.

a. Centralized Integrated System: A system which should have the capacity to update Indicators periodically with their shelf-life and generate indicator as well as district wise rankings, vertical and horizontal comparisons, trends with forecasting, export time-series data of selected variables and maintain keys for integrations with other systems. For all updates, data may be collected through Android-App and synchronized with a system or proforma manually filled \& which further digitized on the system. It is pertinent to highlight that during the annual school census almost all indicators' data updated, if any indicator missing that may be incorporated, if shelf-life of any indicators expired that also be updated with its periodicity, for instance, shelf-life of date of birth of a teacher is a lifetime, however, teacher as well as student attendance, maybe updated periodically and regularly, therefore, the system should have the capability to updated variable before its expiry, and then generate the inconsistencies (detail mentioned below) in the data for further correction so that the planners and decisionmakers can have concurrent $\&$ accurate data, trends, and theme wise reports for special focus.

b. Built in Data (in)-Consistency Check: The aforementioned cases may be tagged as " $\mathrm{A}$ ", "B", " $\mathrm{C}$ " and so on, subsequently coding of the system in such a way so that after digitization data, the system built-in checks will execute and generate school wise list of anomalies that need further verification from either filed or the data provider. The system will ensure that all fields being entered / edited / assembled do not contradict entries that have been previously entered.

\section{References:}

Ashan M. 2003. "An analytical review of Pakistani education policies and plans" Research Papers in Education 18:3, 259-280 DOI:10.1080/0267152032000107329.

Ministry of Federal Education and Professional Training, Government of Pakistan. National Education Policy 2017. Policy, Islamabad: Government of Pakistan, 2018.

William Easterly (2001), "The Political Economy of Growth Without Development: A Case Study of Pakistan." Paper for the Analytical Narratives of Growth Project, Kennedy School of Government, Harvard University.

Mr. Sheraz Ali. PhD Proposal of the lead author: "Enhancing Education Access to Girls: Policies, Barriers, and Impact in Sindh" IBA-University of Sindh Jamshoro (unpublished),2018.

Sindh-EMIS, S. E. (2012). Sindh Education Profile 2011-12. Karachi: Reform Support Unit (RSU), School Education \& Literacy Department (E\&LD) Government of Sindh (GoS).

Sindh-EMIS, S. E. (2013). Sindh Education Profile 2012-13. Karachi: Reform Support Unit (RSU), School Education \& Literacy Department (E\&LD) Government of Sindh (GoS). 